\title{
Pitfalls of PACNS: A Rare Case of Central Nervous System Vasculitis Associated with Toxoplasmosis
}

HUA-XIA YANG, MD, Department of Rheumatology and Clinical Immunology, Peking Union Medical College Hospital, Clinical Immunology Center, Chinese Academy of Medical Sciences and Peking Union Medical College, and the Ministry of Education Key Laboratory, Beijing; HONG-RUI LIU, MD, Department of Pathology, Peking Union Medical College Hospital, Chinese Academy of Medical Sciences and Peking Union Medical College, Beijing; FENG-CHUN ZHANG, MD, Department of Rheumatology and Clinical Immunology, Peking Union Medical College Hospital, Clinical Immunology Center, Chinese Academy of Medical Sciences and Peking Union Medical College, and the Ministry of Education Key Laboratory, Beijing, China. Address correspondence to Dr. F.C. Zhang, Department of Rheumatology and Clinical Immunology, Peking Union Medical College Hospital, Clinical Immunology Center, Chinese Academy of Medical Sciences, No. 1 Shuaifuyuan, Dongcheng District, Beijing 100730, China. E-mail: zhangfccra@aliyun.com. Approved by the Ethics Committees of Peking Union Medical College Hospital (approval number S-K179). J Rheumatol 2017;44:1290-1; doi:10.3899/jrheum.161472

Diagnosis of primary angiitis of the central nervous system (PACNS) is a challenge for clinicians owing to the rarity of the disease and the lack of specific tests ${ }^{1}$. A targeted biopsy is recommended to maximize diagnostic potential ${ }^{2}$.
A 31-year-old worker from South China was admitted with a 7-year history of paroxysmal seizures with loss of consciousness and limb convulsions. He had a habit of eating lamb kebabs and reported no contact with pets. Laboratory
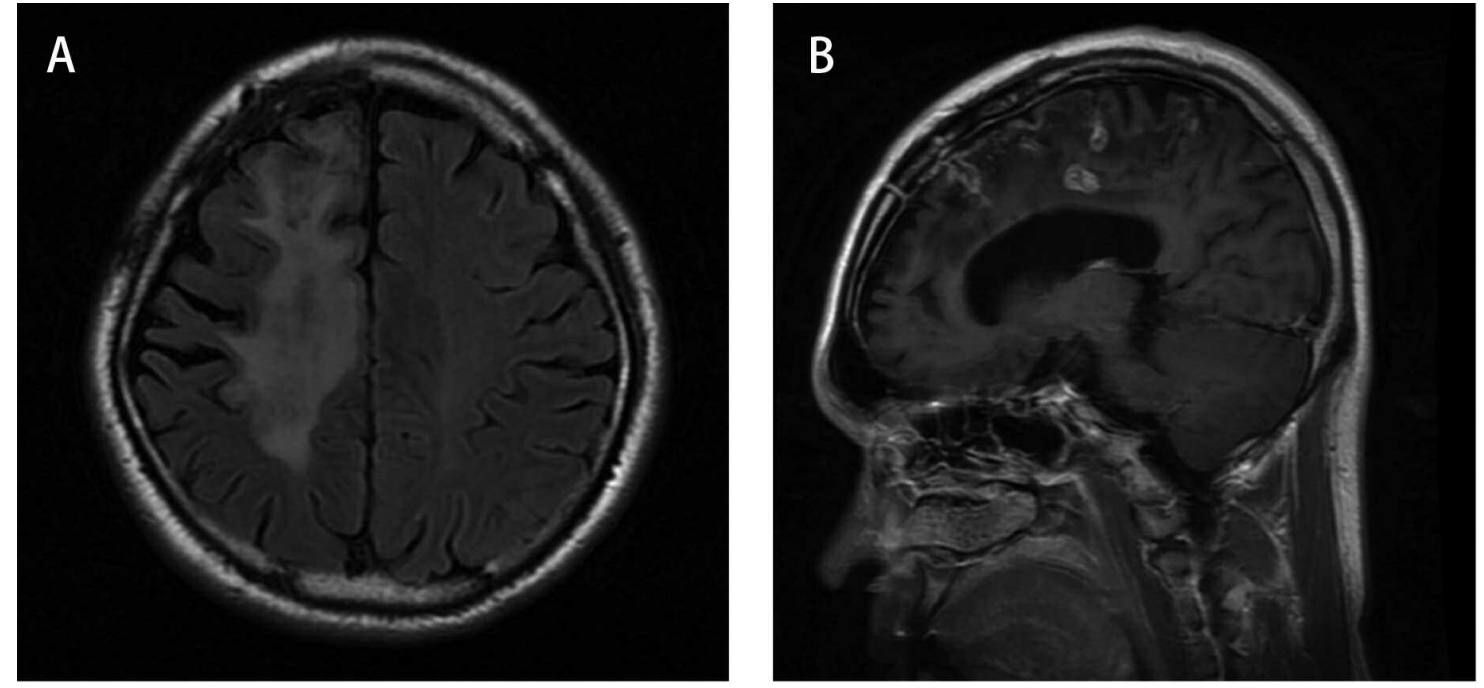

Figure 1. Brain magnetic resonance imaging. A. Extensive lesions restricted to the right cerebral hemisphere on fluid attenuated inversion recovery. B. Multiple foci of nodular and ring enhancement on T1 postcontrast imaging.
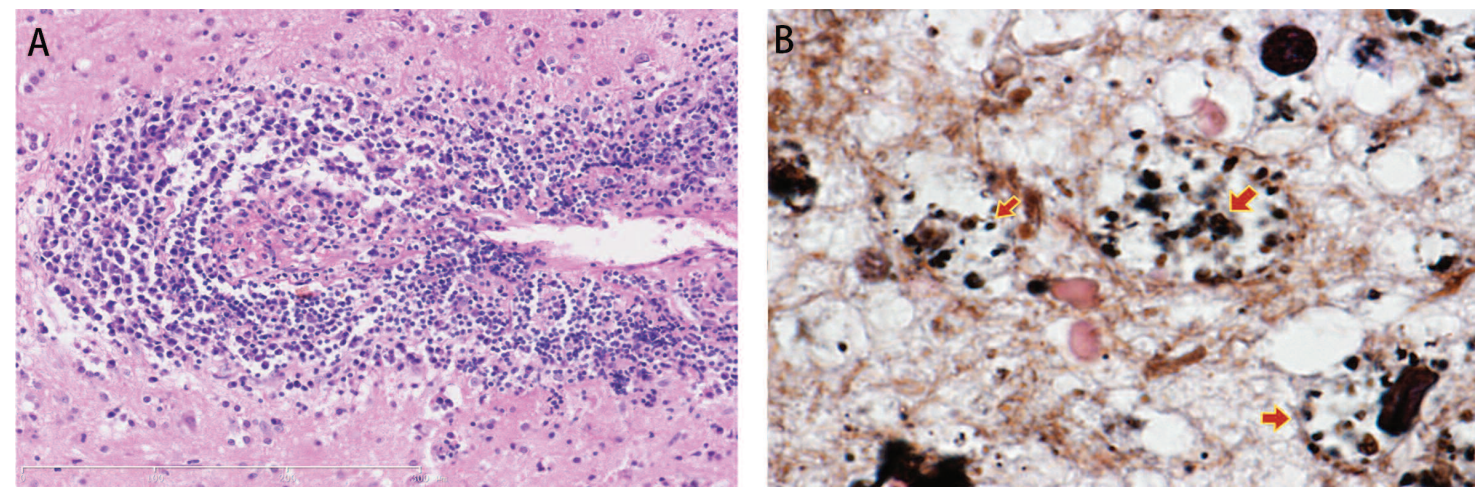

Figure 2. Vasculitis and Toxoplasma gondii in the brain tissue. A. Vasculitis: infiltration of lymphocytic inflammation in and around the vessel wall with large amounts of plasma cells in the brain (H\&E). B. T. gondii: thin-walled cysts containing numerous black and oval to round particles (arrows; black dots, Gomori methenamine-silver staining).

Personal non-commercial use only. The Journal of Rheumatology Copyright (C) 2017. All rights reserved. 
findings showed a slightly elevated erythrocyte sedimentation rate $(28 \mathrm{~mm} / \mathrm{h})$ and negative results from tests for human immunodeficiency virus, antinuclear antibody, and antineutrophil cytoplasmic antibodies. Magnetic resonance imaging of the brain demonstrated multifocal and migrate lesions constricted in the unilateral hemisphere and ring enhancement on T1 postcontrast imaging (Figure 1). The angiography was negative. A targeted resection of the temporal lobe lesions revealed destructive vasculitis with lymphocytic inflammation and fibrinoid necrosis. PACNS was suspected. However, a large dose of prednisone did not result in complete remission. A repeat examination of the biopsy specimen using both $\mathrm{H} \& \mathrm{E}$ and Gomori methenaminesilver stain identified many cysts containing small oval to round objects consistent with toxoplasmosis. Plasma cell infiltration was seen around vessel walls (Figure 2). Toxoplasma-specific IgG was detected in both serum and cerebrospinal fluid. A diagnosis of CNS vasculitis in association with toxoplasmosis was made and the patient responded well to antibiotic therapy.

We report the first documented case, to our knowledge, of CNS vasculitis associated with toxoplasmosis, in a patient with no known immunocompromise. Toxoplasmosis needs to be considered even in non-immunocompromised patients.

\section{REFERENCES}

1. Berlit P, Kraemer M. Cerebral vasculitis in adults: what are the steps in order to establish the diagnosis? Red flags and pitfalls. Clin Exp Immunol 2014;175:419-24.

2. Hajj-Ali RA, Calabrese LH. Diagnosis and classification of central nervous system vasculitis. J Autoimmun 2014;48-49:149-52. 